\title{
Membership, binarity, and rotation of red dwarfs in the nearby open cluster Coma Berenices (Melotte 111)
}

\author{
J.-C. Mermilliod ${ }^{1}$, M. Grenon ${ }^{2}$, and M. Mayor ${ }^{2}$ \\ 1 Laboratoire d'Astrophysique, École polytechnique fédérale de Lausanne (EPFL), Observatoire de Sauverny, 1290 Versoix, \\ Switzerland \\ e-mail: Jean-Claude.Mermilliod@epfl.ch \\ 2 Observatoire de Genève, 1290 Sauverny, Switzerland
}

Received 22 May 2008 / Accepted 22 August 2008

ABSTRACT

\begin{abstract}
Context. Although several attempts have been made to identify solar-type members on the main sequence of the nearby open cluster Coma Berenices (Mel 111), the population of the lower main sequence is still poorly known.

Aims. We observed 46 new candidates to search for new members and monitored known spectroscopic-binary members to determine orbital parameters.

Methods. We obtained a total of 903 radial-velocity measurements of 69 solar-type stars in the field of Mel 111 with the CORAVEL spectrovelocimeter over 20 years.

Results. Among the 35 stars from Trumpler's list, 23 are members according to their radial velocities and photometry. We were able to confirm the membership of only 8 stars among the 46 candidates observed. Six double-lined and ten single-lined spectroscopic binaries were discovered. Six only are members and an orbit was determined for them and for 4 double-lined non-members. The binary frequency is $22 \%$ (7/32). The cluster mean radial velocity is $+0.01 \pm 0.08 \mathrm{~km} \mathrm{~s}^{-1}$ based on 28 members.

Conclusions. The lower main sequence of the Coma Berenices open cluster is still rather poorly populated. The cluster size may be much larger that usually accepted. Accordingly extensive programmes to determine precise proper motions, radial velocities and photometry should be undertaken to identify faint cluster members outside the cluster central area. If a significant population of faint members cannot be identified, Coma Ber could be a prominent example of dynamical evolution leading to star evaporation.
\end{abstract}

Key words. Galaxy: open clusters and associations: individual: Coma Berenices (Melotte 111)- stars: binaries: spectroscopic technique: radial velocities

\section{Introduction}

Coma Berenices (Mel 111) is a nearby, poorly-populated open cluster located in the direction of the North Galactic pole at $\alpha=12^{\mathrm{h}} 25^{\mathrm{m}} \cdot 1$ and $\delta=+26^{\circ} 06^{\prime}(\mathrm{J} 2000)$ at a distance of $85 \mathrm{pc}$ for a diameter estimated as about $5^{\circ}$. Its age is close to that of the Hyades $(\log t=8.65,445 \mathrm{Myr})$, but its metallicity is slightly less than solar, $[\mathrm{Fe} / \mathrm{H}]=-0.05$ (Gratton 2000). The upper main sequence contains a number of bright stars, while the lower main sequence is poorly populated for $V>10.5$. The Coma Berenices cluster is therefore considered as a poorly-populated cluster. Several attempts to discover additional faint members were not successful, as described below.

The bright upper-main-sequence stars, which present Ap and Am stars, were intensively observed, but the solar-type stars received less attention. The lower main sequence is unusually short because it terminates at about $V \sim 10.5$, according to the classical $U B V$ paper of Johnson \& Knuckles (1955) who observed stars from Trumpler's (1938) list of members. This limit corresponds to the spectral type K0V.

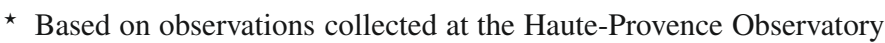
(France).

$\star \star$ Table 1 is also available, and Full Table 8 only available in electronic form at the CDS via anonymous ftp to

cdsarc.u-strasbg.fr $(130.79 .128 .5)$ or via

http://cdsweb.u-strasbg.fr/cgi-bin/qcat?J/A+A/491/951
}

A search for fainter, redder members was performed by de Luca \& Weis (1981), but without better success than Argue \& Kenworthy (1969) who used $B V$ photographic photometry. Bounatiro (1993) proposed a few new members, three of which are included in the present study (Bou 38, 49 and 50). Randich et al. (1996) detected X-ray fluxes of almost all late-F and $\mathrm{G}$ stars in ROSAT PSPC observations. Optical follow-up of twelve X-ray candidate stars (García Lopez et al. 2000) did not confirm any new members fainter than $V=11.0$.

Odenkirchen et al. (1998) investigated the radial structure of the cluster and proposed a number of candidates brighter than $V=10.5$ according to proper-motion criteria. Casewell et al. (2006) produced a list of 60 candidate members with masses between 1.0 and $0.27 M_{\odot}$ selected from proper motions and 2MASS photometry within a circle of $4^{\circ}$ in radius centered on the cluster. We have ten stars in common. Finally, Kraus $\&$ Hillenbrand (2007) used archival data and propose a list of 98 candidate members with probability $>80 \%$, among which 61 are newly identified as high-probability candidates.

We conducted a search for new cluster members and observed 46 candidates. We found only 8 stars presenting a radial velocity in agreement with the Coma Ber mean value and fulfilling the three membership criteria.

Radial velocities with precisions comparable to that of CORAVEL were obtained by Kraft (1965), Jeffries (1999) and Ford et al. (2001). However, no long term monitoring of this sample has been carried out so far and no orbital elements 
have been published for the spectroscopic binaries discovered in Coma Berenices by Trumpler (1938) or Kraft (1965), with the exception of that for Tr 111 (Kraft 1965).

The chemical composition of $\mathrm{A}$ and $\mathrm{F}$ dwarf members were determined by Gebran et al. (2008) who found $[\mathrm{Fe} / \mathrm{H}]=0.07 \pm$ 0.09 dex, slightly higher that the metallicity derived by Friel \& Boesgaard (1992). Kraft (1965) and Ford et al. (2001) published $V \sin i$ values for a number of solar-type stars. New values of $V \sin i$ with a precision of $1 \mathrm{~km} \mathrm{~s}^{-1}$ were also determined from CORAVEL correlation functions.

\section{Observations}

The initial sample of solar-type members in the Coma Ber cluster was taken from the paper of Johnson \& Knuckles (1955) who made their own selection according to the paper of Trumpler (1938), who provided membership information deduced from proper motion, photometry, and radial velocity in the central part of the Coma cluster. Reexamination of Trumpler's table showed that he did not include a few stars in the member list because of the lack of radial velocities. Therefore, we added $\operatorname{Tr} 6,12,35$, 120, 25a, A20, and B1 to the observing list, as well as $\operatorname{Tr} 48$, 142, and 147 suggested later by Olsen (1984).

A few additional stars were also selected from Argue's (1963) $U B V$ data of 180 objects in the Coma Ber region, for example, Argue 8-21, 9-16, and 19-28, also suggested by Olsen. Four stars were selected from Table 1 of Odenkirchen et al. (1998) (HIP 61 205, 62 763, 62 805, 63 493) and one from their Table 2 (HD 106293).

As Melotte 111 is a poor cluster, an extensive search for additional members was undertaken. The literature, catalogues and lists of stars in the region of the North galactic pole were searched for stars located within the limits of the Coma Ber cluster. Few stars with photometric data and/or radial velocities in agreement with membership were found.

The two papers by Malmquist $(1927,1936)$ were the best sources of candidates. He determined magnitudes and colours which permitted us to select stars on the basis of their position in the colour-magnitude diagram. The candidates retained are listed in Table 1 with numbers larger than 413. Table 1 gives for stars not in Trumpler's main catalogue (Trumpler's appendices A and B, and candidate stars), J2000 coordinates from SIMBAD or 2MASS, and cross-references for star designations in several catalogues or lists in the Coma Berenices region.

Finally, five more stars were also observed, HD 105085 , HD 106293 , HD 107 512, HD 111812 and BD +362278.

Three stars ( $\operatorname{Tr} 87,111$ and $25 \mathrm{a}$ ) did not show any correlation dip with CORAVEL. Tr 111 is a double-lined spectroscopic binary with a period shorter than one day and the lack of correlation was expected due to the rapid rotation resulting from the short orbital period.

Five Am star members of Coma Ber (Tr 62, 139, 144, 145 and 183) produced well-defined correlation functions and were also observed to improve the orbits of $\operatorname{Tr} 144$ (Harper 1927) and $\operatorname{Tr} 145$ (Conti \& Barker 1973) available in 1978. We also searched for radial-velocity variations in $\operatorname{Tr} 62$ and 183. These stars are discussed in Sect. 4.

\subsection{Coravel observations}

The observations were obtained with the CORAVEL radialvelocity scanner (Baranne et al. 1979) installed on the Swiss 1-m telescope at the Haute-Provence Observatory, France (OHP) for stars later than spectral type F5 and brighter than $B=12.5$. Between May 1977 and December 1997, eleven to sixteen observations per star were obtained for Trumpler's members. Three to eight measurements were secured for candidates. Binaries were observed more often to derive orbital elements. Radial velocities were determined as usual by fitting a Gaussian curve to the correlation function, or two Gaussians in the cases of blends (for doubled-lined binaries).

The radial velocities are in the system defined by Udry et al. (1999) from high-precision radial-velocities obtained with the ELODIE spectrograph. This calibration corrects for most systematic effects of the CORAVEL system, concerning the dependence of the zero-point shift as a function of the stellar temperature. The integration times ranged from about 200 to as much as $1500 \mathrm{~s}$, the average being about $360 \mathrm{~s}$. The errors on well-exposed individual measurements usually are lower than $0.5 \mathrm{~km} \mathrm{~s}^{-1}$. However, for a few stars with more rapid rotation, the errors may reach $1 \mathrm{~km} \mathrm{~s}^{-1}$.

Projected rotational velocities $(V \sin i$ ) were derived from the width of the correlation functions, following the outline by Benz \& Mayor (1984).

Individual radial velocities will be published in a comprehensive catalogue of 7200 CORAVEL observations of 1253 solar-type dwarfs in nearby open clusters (Mermilliod et al. 2008).

\subsection{Mean radial velocities}

Weighted mean radial velocities were computed, with weights of individual observations taken as $1 / \epsilon^{2}$. The general information and mean radial-velocity are given for the members (Table 2) and non-members (Table 3) separately. $U B V$ magnitudes and colours and spectral types are taken from WEBDA.

The distribution of the mean radial velocities (Fig. 1) shows a clear peak around $V_{\mathrm{r}}=0$ due to the cluster members. It contrasts with the large range covered by the velocities of the stars classified as field stars.

\section{Results}

\subsection{Membership}

The separation between members and non-members was done on the basis of the radial velocities and photometry. The Coma Ber mean radial velocity is close to $0 \mathrm{~km} \mathrm{~s}^{-1}$. Accordingly stars with $-2<V_{\mathrm{r}}<+2 \mathrm{~km} \mathrm{~s}^{-1}$ were selected as candidate members and their positions in the $(V, B-V)$ diagram were examined. Stars falling below the main sequence (MS) were rejected from membership. This concerns only one star, \#293 ( $\left.V_{\mathrm{r}}=-1.31 \mathrm{~km} \mathrm{~s}^{-1}\right)$ because all other non-members have radial velocities well outside these limits, which provides a clear membership criterion. Conversely, the position on the MS or within the MS band is not a sufficient criterion as shown by the $(V, B-V)$ colour-magnitude diagram for the whole sample (Fig. 2).

Although the photometric selection was quite pertinent, we are able to add or confirm very few new members on the lower main sequence. Several stars located on the single-star sequence proved to be non-members according to their radial velocities.

We are however able to confirm the membership of several stars from Trumpler's list: $\operatorname{Tr} 12,48,120$, A20. They were not previously considered by Trumpler because he did not obtain radial velocities to decide on their membership, or because the radial velocity was off the cluster mean, which is the case of $\operatorname{Tr} 120$, a newly discovered binary. 
Table 1. J2000 coordinates and cross-references for candidate stars.

\begin{tabular}{|c|c|c|c|c|c|c|c|c|c|}
\hline No. & RA & Dec & Malm & Arty & Upgren & Argue & Abad & $\mathrm{BD}$ & Other \\
\hline 203 & 121916.26 & +252610.5 & 25.087 & 44 & 26.082 & & 1038 & & Tr A3 \\
\hline 213 & 122405.72 & +260743.1 & 26.170 & & & & 1431 & $+26^{\circ} 2342$ & $\operatorname{Tr} \mathrm{A} 13$ \\
\hline 214 & 122417.15 & +241928.3 & 24.148 & 349 & & & 1444 & $+25^{\circ} 2502$ & Tr A14, HIP 60 511, J122417.15+241928.4 \\
\hline 219 & 122752.66 & +250544.7 & 25.198 & 603 & 25.073 & & 1748 & & $\operatorname{Tr}$ A19 \\
\hline 220 & 122856.43 & +263257.2 & 27.218 & 648 & 27.108 & & 1826 & $+27^{\circ} 2139$ & $\operatorname{Tr}$ A20, J122856.43+263257.4 \\
\hline 221 & 123014.10 & +250142.3 & 25.230 & & 25.078 & & 1924 & & Tr A21, HIP 61012 \\
\hline 222 & 122005.71 & +255436.8 & 26.101 & 72 & 26.087 & $11-14$ & 1097 & & $\operatorname{Tr} \mathrm{B} 1$ \\
\hline 275 & 121851.03 & +261924.4 & 26.075 & 20 & 27.084 & $8-21$ & 995 & $+27^{\circ} 2113$ & \\
\hline 280 & 121905.18 & +261104.4 & 26.084 & & 26.080 & $9-10$ & 1019 & & \\
\hline 282 & 122115.61 & +260914.0 & 26.121 & 132 & 26.092 & $9-16$ & 1183 & & $\mathrm{~J} 122115.63+260914.1$ \\
\hline 293 & 121858.70 & +260330.9 & 26.079 & & 26.077 & $10-21$ & 1009 & & \\
\hline 299 & 122422.95 & +255456.2 & 26.175 & & & $11-24$ & 1453 & & \\
\hline 311 & 122240.65 & +254011.9 & 26.141 & & & $13-18$ & 1297 & & \\
\hline 334 & 122225.82 & +251903.3 & 25.129 & & 26.093 & $15-16$ & 1278 & & \\
\hline 351 & 121908.52 & +250311.6 & 25.084 & & 25.053 & $17-14$ & 1023 & & \\
\hline 379 & 122726.28 & +244657.0 & 25.194 & 577 & 25.071 & $19-28$ & & & \\
\hline 386 & 121343.90 & +225316.7 & 23.039 & & & & 589 & $+23^{\circ} 2433$ & HD 106293 , Bou 38 \\
\hline 390 & 125211.61 & +252224.6 & 25.449 & & & & & $+26^{\circ} 2402$ & HD 111 878, HIP 62805 \\
\hline 395 & 123231.07 & +351952.3 & 35.177 & & & & & $+36^{\circ} 2278$ & HIP 61205 , Bou 50 \\
\hline 399 & 125141.92 & +273226.5 & 28.448 & & & & & $+28^{\circ} 2156$ & HD 111812 , HIP 62763 , LS Com \\
\hline 400 & 130035.18 & +233906.3 & & & & & & $+24^{\circ} 2522$ & HD 113 037, HIP 63493 \\
\hline 414 & 123446.91 & +240937.9 & 24.292 & 993 & & & 2248 & & $\mathrm{~J} 123446.93+240937.7$ \\
\hline 415 & 123652.49 & +240127.1 & 24.314 & 1053 & & & & & \\
\hline 416 & 121649.44 & +245121.1 & 25.055 & & 25.044 & & 833 & $+25^{\circ} 2479$ & \\
\hline 417 & 122259.42 & +245858.4 & 25.141 & 245 & 25.058 & & 1332 & & \\
\hline 418 & 121857.26 & +255310.9 & 26.078 & 23 & 26.076 & & 1003 & & \\
\hline 420 & 122328.17 & +255338.8 & 26.155 & 278 & & & & & $\mathrm{~J} 122328.21+255339.9$ \\
\hline 421 & 122651.04 & +261602.5 & 26.215 & 537 & 27.101 & $8-06$ & 1660 & & $\mathrm{~J} 122651.03+261601.9$ \\
\hline 422 & 122944.66 & +253235.5 & 26.250 & 686 & 26.107 & & 1895 & & \\
\hline 423 & 123330.19 & +261000.2 & 26.286 & 908 & 26.115 & & 2166 & & $\mathrm{~J} 123330.19+261000.1$ \\
\hline 424 & 123855.36 & +255338.1 & 26.364 & & 26.126 & & & & \\
\hline 425 & 124104.97 & +254217.9 & 26.392 & & 26.130 & & 2406 & $+26^{\circ} 2379$ & \\
\hline 427 & 123354.22 & +270804.7 & 27.297 & 938 & 27.121 & & 2194 & & \\
\hline 428 & 125125.12 & +264708.7 & 27.506 & & & & & & \\
\hline 430 & 121839.65 & +274558.8 & 28.108 & 101 & 28.068 & & & & \\
\hline 431 & 123300.68 & +274245.1 & 28.277 & 877 & 28.096 & & 2129 & & $\mathrm{~J} 123300.62+274244.8$ \\
\hline 432 & 123945.90 & +28 1756.7 & 28.345 & & 29.129 & & & & \\
\hline 433 & 124013.00 & +281321.0 & 28.351 & & & & 2395 & $+29^{\circ} 2313$ & HD 110195 \\
\hline 434 & 122825.56 & +295354.4 & 30.178 & & 30.077 & & & $+30^{\circ} 2276$ & \\
\hline 435 & 122827.51 & +295242.3 & 30.179 & & 30.078 & & & $+30^{\circ} 2277$ & \\
\hline 436 & 123500.33 & +301133.6 & 30.252 & & & & & $+30^{\circ} 2296$ & HIP 61409 \\
\hline 437 & 122120.91 & +321035.2 & 32.078 & & & & & $+32^{\circ} 2236$ & HD 107 512, HIP 60 256, Bou 49 \\
\hline
\end{tabular}

Note: this table displays the star numbers according to the numbering system used in WEBDA (http://www . univie.ac.at/webda/) or extends it (No > 413), J2000 coordinates from SIMBAD or 2MASS, cross-references with the lists of Malmquist (1927, 1936), Artjukhina (1955), Upgren (1962), Argue (1963) and Abad \& Vicente (1999). BD refers, as usual, to the Bonner Durchmusterung. Tr A3, A13, A14, A19, A20, A21 and Tr B1 refer to Trumpler's (1938) appendices A and B respectively. J designation are from Casewell et al. (2006). "Bou" refers to Bounatiro (1993). Miscellanous identifications are: HD for the Henry Draper catalogue and HIP for the HIPPARCOS catalogue.

Stars 220 (Tr A20), 282, 420, 421 and 431, confirmed members from our radial velocities, belong to the list of possible members of Casewell et al. (2006) who give membership probabilities of greater than 64\%. In addition, J122706.26+265044.5, identical to $\operatorname{Tr} 132$, is also a true cluster member.

Conversely, several stars from the Casewell et al. (2006) list of candidate members, namely J122417.15+241928.4 (\#214), J123446.93+240937.7 (\#414), J121857.27+255311.1 (\#418) and J123330.19+261000.1 (\#423) are obviously non-members, as is the case for J123814.94+262128.1 (\#199).

The membership of four stars from Table 1 of Odenkirchen et al. (1998), namely \#390, 395, 399 and 400, selected from HIPPARCOS astrometry is confirmed with the present radial velocities. However star \#386 (HD 106293) is probably a nonmember. The large rotation makes the radial velocity less precise and it would be interesting to obtain further data to settle its membership more conclusively. The radial velocities for $\operatorname{Tr} 65$ and 102, two stars also listed in Table 2 of Odenkirchen et al. (1998), fully confirm their membership. These two objects did not obtain the maximum rating in Trumpler's (1938) paper for the radial-velocity criterion.

Conversely, stars $\operatorname{Tr} 35,74,147,148$, A3, A14, A19, A21 and B1 have velocities which clearly indicate non-membership. Star $\operatorname{Tr} 35$, a double-lined binary, lies slightly below the MS, which cannot support its membership. If a member, one would expect to find Tr 35 about $0.7 \mathrm{mag}$ above the MS, according to the mass ratio. It is therefore more distant from the Sun than the cluster. Its systemic velocity is slightly off the mean cluster value. 
Table 2. Mean radial and rotational velocities for 31 member stars.

\begin{tabular}{|c|c|c|c|c|c|c|c|c|c|c|c|c|c|c|c|c|c|}
\hline No. & $V$ & $U-B$ & $B-V$ & $V-I$ & SpT & $V_{\mathrm{r}}$ & $\epsilon$ & $N$ & $\Delta T$ & $P\left(\chi^{2}\right)$ & $V \sin i$ & $\mathrm{rms}$ & $R V_{\text {lit }}$ & Err & $n$ & Src & Rem \\
\hline 12 & 9.54 & & 0.60 & & G0 & +0.55 & 0.15 & 11 & 6934 & 0.195 & 6.6 & 0.6 & +0.7 & 0.8 & 1 & $\mathrm{~b}$ & \\
\hline 19 & 8.12 & -0.02 & 0.39 & 0.477 & F5 V & +0.69 & 0.29 & 13 & 7536 & 0.069 & 21.7 & 2.2 & -0.1 & 0.6 & 3 & $\mathrm{a}$ & \\
\hline 36 & 8.12 & -0.04 & 0.40 & 0.482 & F3 V & +0.03 & 0.79 & 12 & 5820 & 0.009 & 35.1 & 3.5 & +0.7 & 1.8 & 3 & $\mathrm{a}$ & \\
\hline 48 & 8.80 & 0.08 & 0.52 & & F7 V & -0.43 & 0.14 & 37 & 3565 & 0.000 & 9.6 & 1.4 & & & & & SB2O \\
\hline 53 & 8.74 & 0.02 & 0.51 & & F7 V & -0.37 & 0.11 & 38 & 5852 & 0.000 & 6.1 & 0.4 & -9.7 & 0.2 & 1 & $\mathrm{a}$ & SB1O \\
\hline 58 & 8.82 & -0.00 & 0.50 & & F7 V & +0.40 & 0.16 & 15 & 7271 & 0.441 & 16.0 & 0.4 & +1.5 & 0.1 & 1 & $\mathrm{a}$ & \\
\hline 65 & 9.02 & 0.04 & 0.57 & 0.673 & F5 V & -0.51 & 0.12 & 16 & 7271 & 0.754 & 9.5 & 0.5 & -2.4 & 1.0 & 1 & $\mathrm{~d}$ & \\
\hline 76 & 9.31 & 0.08 & 0.59 & 0.631 & G0 V & -0.75 & 0.12 & 13 & 7271 & 0.769 & 9.0 & 0.4 & +0.3 & 1.0 & 1 & $\mathrm{~d}$ & \\
\hline 85 & 9.31 & 0.06 & 0.59 & 0.653 & G0 V & +0.15 & 0.12 & 11 & 7271 & 0.976 & 7.8 & 0.5 & +2.6 & 1.0 & 1 & $\mathrm{~d}$ & \\
\hline 86 & 8.53 & -0.02 & 0.46 & 0.544 & F7 V & -0.77 & 0.21 & 13 & 7536 & 0.646 & 20.7 & 2.1 & +1.3 & 0.9 & 2 & $\mathrm{a}$ & \\
\hline 90 & 8.55 & -0.04 & 0.46 & 0.542 & F6 V & +0.28 & 0.18 & 13 & 7273 & 0.354 & 15.3 & 0.5 & +0.7 & 0.1 & 1 & $\mathrm{a}$ & \\
\hline 92 & 8.68 & 0.02 & 0.53 & 0.697 & F8 V & -0.23 & 0.15 & 30 & 7540 & 0.305 & 20.8 & 2.1 & -1.5 & 2.1 & 4 & $\mathrm{e}$ & \\
\hline 97 & 9.18 & 0.00 & 0.54 & 0.643 & F8 V & -0.23 & 0.10 & 64 & 4620 & 0.000 & 17.7 & 0.3 & +3.6 & 13.8 & 5 & $\mathrm{e}$ & SB1O \\
\hline 101 & 8.38 & -0.03 & 0.45 & 0.521 & F6 V & -0.16 & 0.42 & 13 & 7273 & 0.034 & 25.3 & 2.5 & -1.2 & 1.3 & 2 & $\mathrm{a}$ & \\
\hline 102 & 9.33 & 0.07 & 0.60 & 0.692 & G0 V & +0.18 & 0.06 & 59 & 6218 & 0.000 & 4.3 & 0.4 & -3.1 & 1.0 & 1 & $\mathrm{c}$ & SB1O \\
\hline 114 & 8.58 & -0.05 & 0.45 & 0.539 & F7 V & +0.77 & 0.18 & 11 & 7272 & 0.974 & 15.5 & 0.4 & -0.7 & 0.1 & 1 & $\mathrm{a}$ & \\
\hline 118 & 8.36 & -0.03 & 0.44 & 0.518 & F6 V & +0.27 & 0.24 & 11 & 7539 & 0.188 & 16.5 & 0.5 & -0.4 & 0.2 & 2 & $\mathrm{a}$ & \\
\hline 120 & 9.76 & 0.28 & 0.80 & & G7 & +1.03 & 0.12 & 26 & 5510 & 0.000 & 6.4 & 0.5 & +12.1 & 1.0 & 1 & $\mathrm{~d}$ & SB1O \\
\hline 132 & 9.88 & 0.18 & 0.67 & 0.728 & G5 V & -0.03 & 0.12 & 11 & 7539 & 0.501 & 3.5 & 0.8 & -0.5 & 1.0 & 1 & $\mathrm{~d}$ & \\
\hline 150 & 9.78 & 0.31 & 0.78 & 0.852 & G9 V & +0.30 & 0.12 & 29 & 4346 & 0.000 & 12.5 & 2.5 & +0.1 & 0.3 & 1 & $\mathrm{a}$ & SB2O \\
\hline 162 & 8.60 & -0.04 & 0.48 & 0.546 & F7 V & -0.04 & 0.22 & 12 & 7537 & 0.256 & 18.3 & 0.5 & -0.5 & 1.0 & 2 & $\mathrm{a}$ & \\
\hline 213 & 10.48 & 0.38 & 0.77 & & $\mathrm{~K} 0 \mathrm{~V}$ & +0.12 & 0.14 & 8 & 5104 & 0.846 & 5.0 & 0.9 & +0.9 & 1.0 & 1 & $\mathrm{c}$ & $\operatorname{Tr}$ A13 \\
\hline 220 & 10.78 & & 0.89 & & K0 III & +0.83 & 0.16 & 6 & 3631 & 0.738 & 3.5 & 1.2 & +0.3 & 0.8 & 1 & $\mathrm{~b}$ & $\operatorname{Tr} \mathrm{A} 20$ \\
\hline 282 & 11.46 & 0.84 & 1.10 & & K1 V & -0.01 & 0.20 & 4 & 2834 & 0.969 & 1.4 & 2.8 & & & & & Argue 9-16 \\
\hline 390 & 8.87 & & & & F8 V & -0.83 & 0.57 & 2 & 2168 & 0.703 & 12.7 & 1.6 & -2.4 & 1.0 & 1 & $\mathrm{c}$ & Griffin \\
\hline 395 & 9.71 & & 0.63 & & G0 & -1.46 & 0.21 & 4 & 2063 & 0.891 & 6.4 & 0.9 & -0.5 & 2.0 & 1 & $\mathrm{c}$ & Bou 50 \\
\hline 400 & 8.24 & & & & F5 V & -0.80 & 0.79 & 2 & 1091 & 0.722 & 23.8 & 6.5 & & & & & Griffin \\
\hline 420 & 12.13 & & 1.20 & & & -0.20 & 0.20 & 5 & 4020 & 0.686 & 4.7 & 1.3 & & & & & Malm 26.155 \\
\hline 421 & 11.94 & & 1.15 & & $\mathrm{~K} 2 \mathrm{~V}$ & -0.69 & 0.25 & 5 & 3688 & 0.153 & 1.0 & 3.6 & & & & & Malm 26.215 \\
\hline 431 & 11.25 & & 1.00 & & K0 IV & -0.10 & 0.18 & 5 & 3697 & 0.846 & 3.8 & 1.9 & & & & & Malm 28.277 \\
\hline 436 & 8.63 & & 0.59 & & F8 V & -1.91 & 0.20 & 8 & 3633 & 0.251 & 14.1 & 0.5 & & & & & Malm 30.252 \\
\hline
\end{tabular}

Notes: stellar identification, according to Trumpler (1938) and extension in WEBDA, $V, B-V, U-B$, and $V-I_{K}$ photometric indices, spectral types, CORAVEL mean radial velocities and errors in $\mathrm{km} \mathrm{s}^{-1}$, the number of observations, probabilit $P\left(\chi^{2}\right)$ that the scatter is due to random errors, projected rotation $(V \sin i)$ and error, also in $\mathrm{km} \mathrm{s}^{-1}$, radial velocities from the literature, their error and sources, and remarks on binarity and cross-identifications. Literature sources: a: Kraft (1965); b: Glushkova (1993); c: Ford et al. (2001); d: Jeffries (1999) ; e: Trumpler (1938).

Finally, most stars selected outside Trumpler's list were found to be non-members. Their radial velocities are significantly different from the cluster mean velocity, which confirms the difficulty of identifying new members. So far this does not mean that the Coma Berenices open cluster does not contain Gand K-type dwarfs, but that a lot of effort is required to find many more members.

\subsection{Mean cluster velocity}

The mean cluster velocity is $\left\langle V_{\mathrm{r}}\right\rangle=0.01 \pm 0.08(0.44 \mathrm{rms})$ from 28 members listed in Table 2 . The observed dispersion is quite small and the radial velocity is therefore a very efficient criterion for membership determination.

\subsection{Spectroscopic binaries}

Trumpler (1938) already detected several spectroscopic binaries, namely Tr 48 (Var?), 97, 102, and 150. Kraft (1965) classified stars $\operatorname{Tr} 53$ and 65 as suspected binaries. Concerning $\operatorname{Tr} 120$, the open-cluster database contains only one radial-velocity measurement, by Jeffries (1999), and no mention of its binary character. Its duplicity became evident in March 1979, because the fifth observation differed by $9 \mathrm{~km} \mathrm{~s}^{-1}$ from the previous measurements.

The six spectroscopic binaries, 2 SB2 and 4 SB1, found among the members were monitored until the number of observations permitted us to compute an orbit. The orbital elements are given in Table 4. Tr 97 and 150 have circular orbits as expected for periods around 3 days. The periods for the other binary members are longer and range from 48 to 444 days. Tr 111 , a double-lined binary with a period as short as 0.96 (Kraft 1965), could not be observed because of the induced rapid rotation and the resulting broad lines. The resulting binary percentage is $22 \%$ (7/32), taking $\operatorname{Tr} 111$ into account.

Seven binaries were detected among the non-members, 4 SB2, 3 SB1, and an orbit was determined for the four doublelined binary stars Tr 35, 147, \#416 (Malm 2555), and 433 (Malm 28 351 ). The orbital elements are also presented in Table 4. We do not support the hypothesis that Tr A21 is a binary. Our mean value, $+30.5 \mathrm{~km} \mathrm{~s}^{-1}$ based on 5 observations agrees closely with the Ford et al. (2001) radial velocity, $+30.6 \mathrm{~km} \mathrm{~s}^{-1}$. Accordingly Tr A21 is neither a binary nor a member.

\subsection{Rotation}

The projected rotational velocities published by Kraft (1965) had a lower resolution at $12 \mathrm{~km} \mathrm{~s}^{-1}$. Ford et al. (2001) obtained a better resolution limit at $6 \mathrm{~km} \mathrm{~s}^{-1}$. CORAVEL observations allow us to determine rotational velocities with a precision of $1 \mathrm{~km} \mathrm{~s}^{-1}$. Figure 13 presents the distribution of the projected rotational velocities as a function of $B-V$.

The two stars with short periods, $\operatorname{Tr} 97$ and especially $\operatorname{Tr} 150$ $\left(B-V=0.78, V \sin i=12.5 \mathrm{~km} \mathrm{~s}^{-1}\right)$, rotate faster than the other 
Table 3. Mean radial and rotational velocities for non-member stars.

\begin{tabular}{|c|c|c|c|c|c|c|c|c|c|c|c|c|}
\hline No. & $V$ & $B-V$ & $U-B$ & SpT & $V_{\mathrm{r}}$ & $\epsilon$ & $n$ & $\Delta T$ & $P\left(\chi^{2}\right)$ & $V \sin i$ & Err & Rem \\
\hline 6 & 8.79 & 0.50 & -0.05 & F9 V & -24.63 & 0.18 & 6 & 3632 & 0.745 & 2.7 & 1.4 & \\
\hline 35 & 8.37 & 0.41 & 0.03 & F6 V & -2.49 & 0.29 & 39 & 1138 & 0.000 & 21.1 & 4.0 & SB2O \\
\hline 74 & 8.56 & 0.56 & & G5 & -6.58 & 0.15 & 8 & 5119 & 0.650 & 7.9 & 0.6 & \\
\hline 127 & 8.80 & 0.54 & 0.04 & $\mathrm{~F} 8 / 9 \mathrm{~V}$ & -19.97 & 0.23 & 8 & 7539 & 0.025 & 7.3 & 0.6 & \\
\hline 135 & 10.10 & 0.91 & 0.60 & G8 III & +21.08 & 0.15 & 6 & 5463 & 0.743 & 3.2 & 1.1 & \\
\hline 142 & 9.96 & 0.65 & 0.13 & G5 & +8.52 & 0.21 & 4 & 2589 & 0.975 & 1.9 & 1.9 & \\
\hline 147 & 8.58 & 0.55 & 0.02 & F6 IV-V & -13.68 & 0.26 & 19 & 3565 & 0.000 & 21.7 & 2.2 & SB2O \\
\hline 203 & 10.79 & 0.87 & 0.57 & G9 V & -14.57 & 0.17 & 5 & 5434 & 0.844 & 0.0 & 0.0 & $\operatorname{Tr} \mathrm{A} 3$ \\
\hline 214 & 10.00 & 0.79 & 0.33 & G9 V & +62.44 & 0.19 & 5 & 5454 & 0.400 & 4.0 & 1.4 & $\operatorname{Tr} \mathrm{A} 14$ \\
\hline 219 & 11.18 & 0.82 & 0.39 & G9 V & -16.26 & 0.20 & 5 & 5789 & 0.713 & 1.9 & 1.8 & $\operatorname{Tr}$ A19 \\
\hline 221 & 10.37 & 0.82 & 0.41 & G7 V & +30.51 & 0.22 & 5 & 5454 & 0.220 & 3.0 & 1.7 & $\operatorname{Tr} \mathrm{A} 21$ \\
\hline 222 & 11.26 & 0.98 & & & -44.59 & 0.15 & 7 & 4363 & 0.880 & 1.1 & 1.5 & Tr B1 \\
\hline 275 & 10.82 & 0.95 & 0.74 & K1 V & -6.92 & 0.87 & 15 & 3564 & 0.000 & 4.5 & 0.6 & Argue $8-21$, SB \\
\hline 280 & 11.49 & 0.90 & 0.57 & G8 III & -4.36 & 0.18 & 5 & 5431 & 0.803 & 1.8 & 1.6 & Argue $9-10$ \\
\hline 293 & 11.70 & 0.94 & 0.55 & G8 III & -1.31 & 0.19 & 5 & 5082 & 0.894 & 3.0 & 1.9 & Argue $10-21$ \\
\hline 299 & 12.38 & 1.14 & 1.21 & & -25.49 & 0.25 & 3 & 3628 & 0.688 & 1.3 & 3.9 & Argue $11-24$ \\
\hline 311 & 10.47 & 0.71 & 0.27 & & -50.24 & 0.29 & 5 & 5454 & 0.049 & 0.8 & 2.6 & Argue 13-18 \\
\hline 334 & 11.61 & 0.88 & 0.54 & G8 III & +13.68 & 0.21 & 4 & 5813 & 0.686 & 2.8 & 0.0 & Argue $15-16$ \\
\hline 351 & 12.33 & 1.00 & 0.57 & K0 & +41.71 & 0.27 & 3 & 3628 & 0.492 & 0.0 & 0.0 & Argue $17-14$ \\
\hline 379 & 11.65 & 1.26 & 1.24 & $\mathrm{~K} 2 \mathrm{~V}$ & -5.73 & 0.70 & 4 & 3183 & 0.000 & 2.3 & 1.8 & Argue $19-28$, SB \\
\hline 386 & 8.08 & 0.41 & & F5 V & +6.83 & 2.29 & 3 & 617 & 0.196 & 66.8 & 7.8 & Bou 38 \\
\hline 414 & 12.01 & & & & +10.18 & 0.24 & 3 & 1455 & 0.390 & 1.9 & 0.0 & Malm 24.292 \\
\hline 415 & 12.18 & & & & -6.80 & 0.95 & 3 & 1455 & 0.000 & 5.1 & 3.3 & Malm 24.314, SB \\
\hline 416 & 10.51 & 0.79 & & G8 III & +12.45 & 5.75 & 5 & 4319 & 0.000 & 1.1 & 0.0 & Malm 25.055, SB2O \\
\hline 417 & 11.32 & 1.05 & & & +79.27 & 0.29 & 4 & 4419 & 0.105 & 0.0 & 0.0 & Malm 25.141 \\
\hline 418 & 11.51 & 1.03 & & G8 III & +75.07 & 0.20 & 4 & 3688 & 0.856 & 2.8 & 0.0 & Malm 26.078 \\
\hline 422 & 11.23 & 1.04 & & K0 III & +14.78 & 0.22 & 4 & 4427 & 0.371 & 2.7 & 0.0 & Malm 26.250 \\
\hline 423 & 11.12 & 1.05 & & K0 III & +74.81 & 2.68 & 4 & 4427 & 0.000 & 0.0 & 0.0 & Malm 26.286, SB \\
\hline 424 & 10.91 & 0.96 & & K3 V & -15.76 & 0.19 & 5 & 5459 & 0.341 & 3.7 & 1.3 & Malm 26.364 \\
\hline 425 & 10.24 & 0.76 & & G5 & +14.54 & 0.17 & 5 & 3982 & 0.395 & 2.1 & 1.6 & Malm 26.392 \\
\hline 427 & 11.72 & 1.08 & & G8 V & +12.36 & 0.22 & 4 & 4401 & 0.652 & 2.6 & 2.3 & Malm 27.297 \\
\hline 428 & 11.74 & & & & +10.09 & 0.55 & 13 & 4366 & 0.000 & 7.9 & 1.6 & Malm 27.506, SB \\
\hline 430 & 11.22 & 0.92 & & K0 III & -30.10 & 0.20 & 4 & 4432 & 0.898 & 1.1 & 2.7 & Malm 28.108 \\
\hline 432 & 11.51 & & & K1 V & +7.66 & 0.25 & 5 & 4340 & 0.126 & 3.1 & 1.6 & Malm 28.345 \\
\hline 433 & 10.13 & & & & -16.20 & 10.50 & 4 & 3980 & 0.000 & 1.6 & 2.2 & Malm 28.351, SB2O \\
\hline 434 & 10.67 & 0.87 & & G8 III & -15.87 & 0.18 & 5 & 3984 & 0.965 & 1.7 & 0.0 & Malm 30.178 \\
\hline 435 & 10.07 & 0.71 & & G5 & -14.86 & 0.48 & 7 & 5100 & 0.000 & 4.8 & 1.7 & Malm 30.179, SB \\
\hline 437 & 9.10 & 0.45 & & G0 & -4.05 & 2.07 & 7 & 2063 & 0.000 & 9.4 & 0.5 & Bou 49, SB \\
\hline
\end{tabular}

Notes: stellar identification, according to Trumpler (1938) and extension in WEBDA, $V, B-V$, and $U-B$ photometric indices, spectral types, CORAVEL mean radial velocities and errors in $\mathrm{km} \mathrm{s}^{-1}$, the number of observations, probability $P\left(\chi^{2}\right)$ that the scatter is due to random errors, projected rotation $(V \sin i)$ and error, also in $\mathrm{km} \mathrm{s}^{-1}$, and remarks on binarity and cross-identifications.

members of the same colour, which is easily explained by the acceleration of the rotation due to circularization of the orbits. The rotation of star $\operatorname{Tr} 120$ appears to be normal for its $B-V$ colour, while the three binaries with periods longer than 17 days seem to rotate slightly slower than the other stars with similar colours. The effect is more marked for $\operatorname{Tr} 53(B-V=0.51, V \sin i=$ $\left.6.1 \mathrm{~km} \mathrm{~s}^{-1}\right)$.

\subsection{Colour-magnitude diagram}

Figure 14 displays the $(V, B-V)$ colour-magnitude diagram for the 31 members of Table 2 . The main sequence seems normally populated for $V<10.5$ and is very sparse at fainter magnitudes. The efforts devoted to search for late G- and K-type members in Coma Berenices were not very successful. The main sequence of Casewell et al. (2006) seems to be more evenly populated, although we showed that several candidates of their Table 2 are non-members (see Sect. 3.1).

Two binaries, $\operatorname{Tr} 120$ and 150, are located on the binary ridge, close to each other. Such a location is expected for the double-lined binary $\operatorname{Tr} 150$, but not for $\operatorname{Tr} 120$ because only one component was observed in the correlation functions, although a secondary nearly as bright as the primary would be expected from the photometry. The minimum mass for the secondary is $M_{2}=0.52$, with $f(m)=0.0699$ and $M=0.90 M_{\odot}$ for the primary.

Star \#436 is also located close to the binary ridge, but does not show any sign of variability, $P\left(\chi^{2}\right)=0.251$, with 8 measures covering a time interval of 3633 days. Two other stars, $\operatorname{Tr} 65$ and 92, are also located above the single-star locus and could be considered as photometrically detected binaries. Although 30 observations were obtained for Tr 92 over a period of 7540 days, no significant variation could be detected, which is reflected by the value of $P\left(\chi^{2}\right)=0.305$. The same is true for Tr 65, with 16 observations over 7271 days and $P\left(\chi^{2}\right)=0.754$. However, according to the depth effect discussed below, they could be at a smaller distance than the cluster centre and perhaps belong to the corona of the cluster rather than to the core.

The lower sequence (continuous curve) plotted in Fig. 14 is fitted to the mode of the star distribution with a distance modulus $m-M=4.65$, corresponding to a distance of $85 \mathrm{pc}$. The scatter 


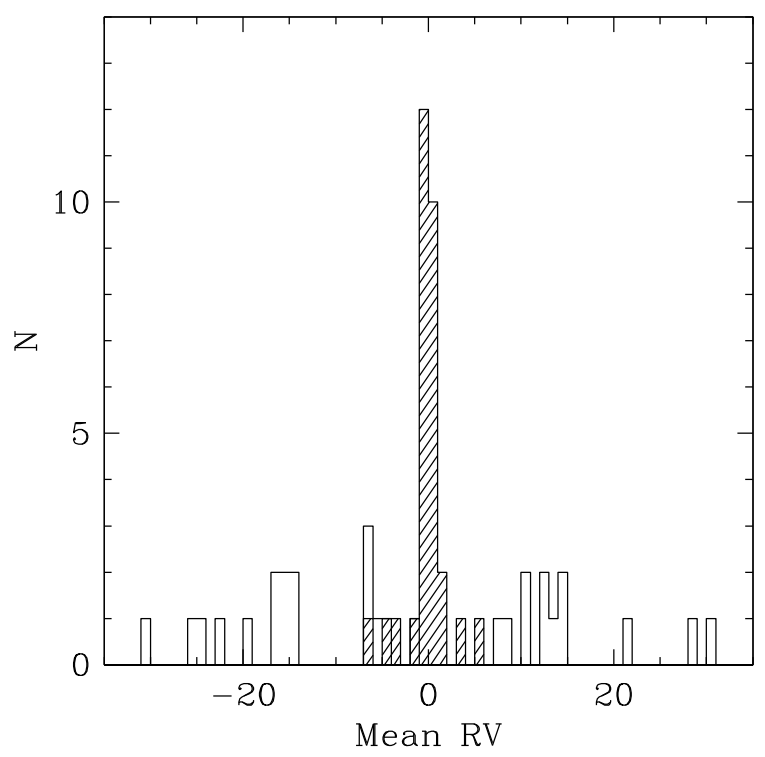

Fig. 1. Distribution of the observed radial velocities. The peak close to $V_{\mathrm{r}}=0$ (hatched histogram) is due to the cluster members.

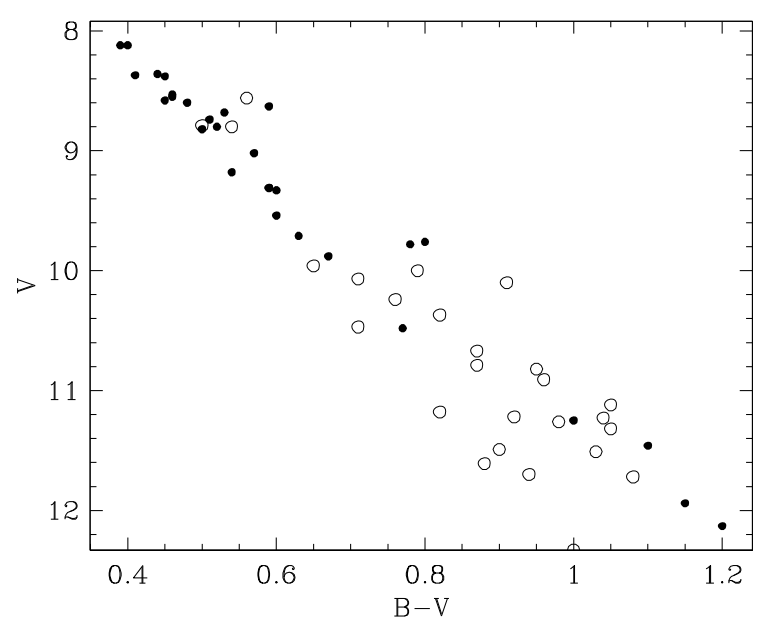

Fig. 2. Colour-magnitude diagram of the whole sample. Members are displayed with filled circles, and non-members with open circles. Several stars selected by the photometry and located closely on the main sequence are in fact non-members.

around this sequence is larger than expected in well-behaved open clusters. This results from the short distance to the cluster and is mainly due to the depth effect, as in the Hyades. If the mean distance is taken as $85 \mathrm{pc}$ and a radius of $5^{\circ}$ is adopted, the linear radius is $7.4 \mathrm{pc}$. Accordingly the closest and farthest distances are 77.6 and $92.4 \mathrm{pc}$, corresponding to distance-moduli of 4.45 and 4.82 respectively. The resulting depth of $0.37 \mathrm{mag}$ matches the effect observed. If a radius of $10^{\circ}$ is used, as would be necessary to take into account probable members located at such a distance from the cluster centre, then the radius is $14.8 \mathrm{pc}$ and the extreme distance-moduli become 4.23 and 4.99 for the closest and most distant edges. Because the cluster proper motion is small and the mean radial velocity nearly zero, no kinematical individual distance can be computed.

\subsection{Candidates from other studies}

Star \#388 (HIP 62384, HD 111154, BD +23 2492, F9 $\mathrm{V}+\mathrm{G} 1.5 \mathrm{~V}$ ), considered as a member from its kinematics

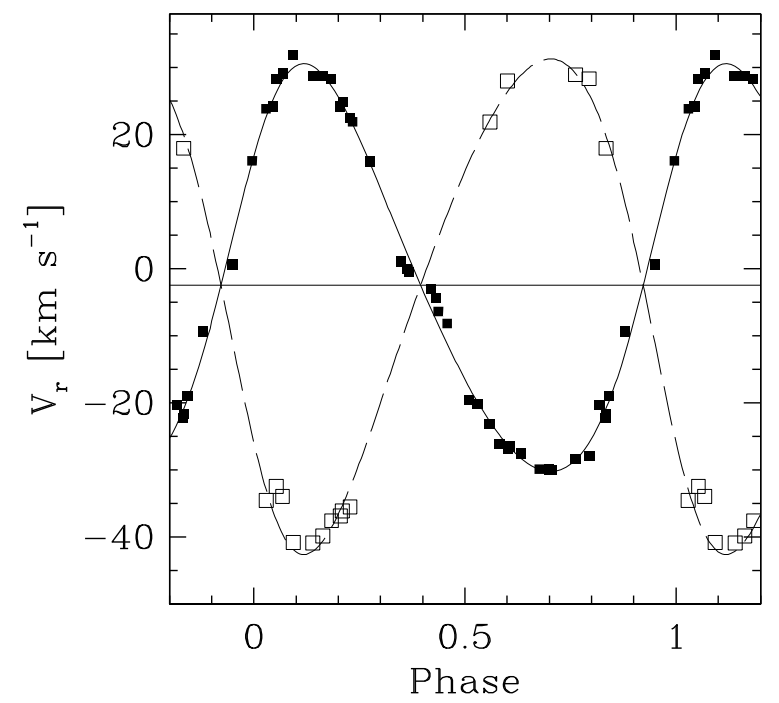

Fig. 3. Radial-velocity curve for the double-lined binary $\operatorname{Tr} 35$.

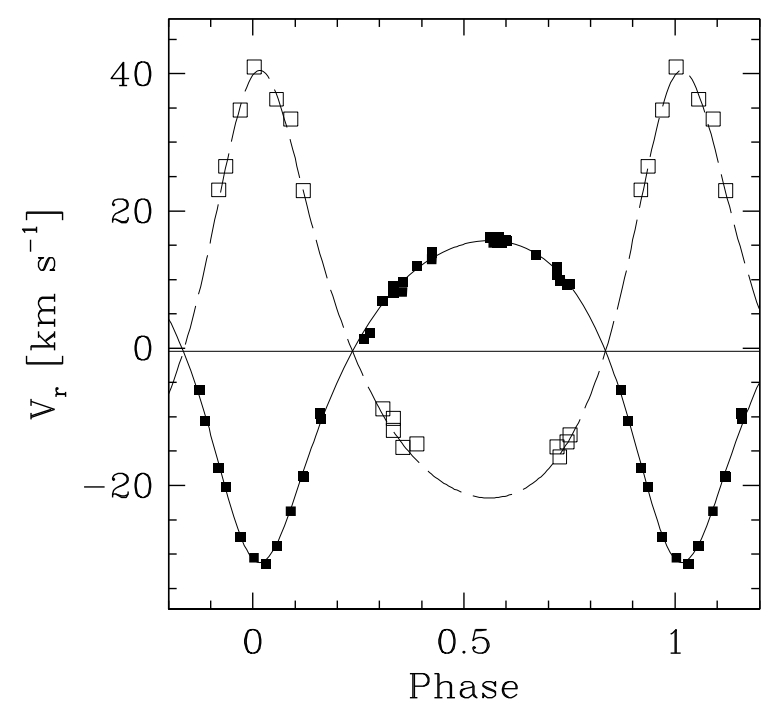

Fig. 4. Radial-velocity curve for the double-lined binary $\operatorname{Tr} 48$.

by Odenkirchen et al. (1998), was observed in radial velocity by Griffin with his spectrograph in Cambridge (Griffin 2001), but also extensively with the CORAVEL at OHP. He found that this star is a double-lined binary and determined the orbital elements. The period is $26^{\mathrm{d}} .971$ and the systemic velocity, $\gamma=+0.55 \mathrm{~km} \mathrm{~s}^{-1}$, confirms the membership of HD 111154 in the Coma Ber cluster. The projected rotational velocities determined from CORAVEL correlation functions are $V \sin i=$ $5.7 \pm 0.5 \mathrm{~km} \mathrm{~s}^{-1}$ for the primary and $V \sin i=3.2 \pm 0.8 \mathrm{~km} \mathrm{~s}^{-1}$ for the secondary (Griffin 2001). Star \#388 is represented by a cross in Fig. 14 at $V=8.40$ and $B-V=0.56$, and lies close to the upper binary ridge in agreement with it beeing a double-lined binary.

Odenkirchen et al. (1998) produced a list of 11 kinematic cluster members with $V>8.5$. The membership of two of them, $\operatorname{Tr} 65$ and 102, is confirmed by our observations. The radial velocities of Ford et al. (2001) support the membership of five other stars from Table 2 of Odenkirchen et al. (1998). The radial velocities and $V$ and $B-V$ photometry are reproduced in Table 5, which gives the WEBDA extended numbering, HD/BD identifications and the parameters taken from Ford et al. (2001). These five stars are represented by crosses in Fig. 14. Four are located 
Table 4. Orbital elements of ten spectroscopic binaries.

\begin{tabular}{|c|c|c|c|c|c|c|c|c|c|c|c|}
\hline No & $\begin{array}{c}P \\
{[\mathrm{~d}]}\end{array}$ & $\begin{array}{c}T \\
\text { [HJD-2 } 400000] \\
\end{array}$ & $\begin{array}{c}\gamma \\
{\left[\mathrm{km} \mathrm{s}^{-1}\right]}\end{array}$ & $e$ & $\begin{array}{c}\omega \\
{\left[{ }^{\circ}\right]}\end{array}$ & $\begin{array}{c}K_{1} \\
{\left[\mathrm{~km} \mathrm{~s}^{-1}\right]}\end{array}$ & $\begin{array}{c}K_{2} \\
{\left[\mathrm{~km} \mathrm{~s}^{-1}\right]}\end{array}$ & $\begin{array}{l}f(m) \\
{\left[M_{\odot}\right]} \\
\end{array}$ & $\begin{array}{l}a \sin i \\
{[\mathrm{Gm}]}\end{array}$ & $\begin{array}{l}\sigma(\mathrm{O}-\mathrm{C}) \\
{\left[\mathrm{km} \mathrm{s}^{-1}\right]}\end{array}$ & $n_{\mathrm{obs}}$ \\
\hline \multirow[t]{2}{*}{35} & 42.582 & 44985.90 & -2.49 & 0.158 & 303.2 & 30.42 & 36.95 & 0.215 & 21.36 & 1.93 & 39 \\
\hline & 0.0103 & 0.57 & 0.29 & 0.013 & 4.6 & 0.42 & 0.76 & 0.015 & 0.49 & & \\
\hline \multirow[t]{2}{*}{48} & 58.7220 & 44994.99 & -0.43 & 0.320 & 347.9 & 23.45 & 31.15 & 0.0668 & 17.94 & 0.87 & 37 \\
\hline & 0.0047 & 0.29 & 0.14 & 0.009 & 1.2 & 0.24 & 0.44 & 0.0027 & 0.24 & & \\
\hline \multirow[t]{2}{*}{53} & 444.19 & 43424.9 & -0.37 & 0.191 & 342.6 & 11.51 & & 0.0665 & 69.0 & 0.56 & 38 \\
\hline & 0.28 & 4.1 & 0.11 & 0.014 & 3.4 & 0.14 & & 0.0030 & 1.1 & & \\
\hline \multirow[t]{2}{*}{97} & 3.023373 & 44999.437 & -0.23 & 0.000 & & 51.39 & & 0.04261 & 2.1365 & 0.79 & 64 \\
\hline & 0.000003 & 0.002 & 0.10 & fixed & & 0.14 & . & 0.00035 & 0.0059 & & \\
\hline \multirow[t]{2}{*}{102} & 48.03078 & 44968.043 & +0.18 & 0.452 & 193.86 & 27.74 & & 0.0755 & 16.34 & 0.41 & 59 \\
\hline & 0.00036 & 0.040 & 0.06 & 0.003 & 0.06 & 0.13 & & 0.0014 & 0.10 & & \\
\hline \multirow[t]{2}{*}{120} & 294.90 & 44732.5 & +1.03 & 0.327 & 246.9 & 13.93 & & 0.0699 & 53.4 & 0.54 & 25 \\
\hline & 0.12 & 1.4 & 0.12 & 0.012 & 2.0 & 0.20 & & 0.0039 & 1.0 & & \\
\hline \multirow[t]{2}{*}{147} & 52.7917 & 45005.28 & -13.68 & 0.479 & 70.1 & 33.94 & 49.27 & 0.1449 & 31.39 & 1.24 & 23 \\
\hline & 0.0049 & 0.37 & 0.26 & 0.008 & 1.2 & 0.32 & 0.61 & 0.0064 & 0.32 & & \\
\hline \multirow[t]{2}{*}{150} & 3.558151 & 45002.746 & +0.30 & 0.000 & & 75.79 & 79.12 & 0.1608 & 3.708 & 0.92 & 29 \\
\hline & 0.000003 & 0.001 & 0.12 & fixed & & 0.22 & 0.25 & 0.0014 & 0.010 & & \\
\hline \multirow[t]{2}{*}{416} & 164.621 & 45069.04 & +18.43 & 0.788 & 210.8 & 25.91 & & 0.069 & 36.1 & 2.14 & 29 \\
\hline & 0.080 & 0.86 & 0.54 & 0.026 & 3.3 & 0.84 & & 0.018 & 3.2 & & \\
\hline \multirow[t]{2}{*}{433} & 17.778507 & 44990.055 & -15.37 & 0.569 & 59.77 & 55.31 & 56.43 & 0.1736 & 11.118 & 0.89 & 30 \\
\hline & 0.000044 & 0.010 & 0.12 & 0.002 & 0.43 & 0.25 & 0.27 & 0.0033 & 0.071 & & \\
\hline
\end{tabular}

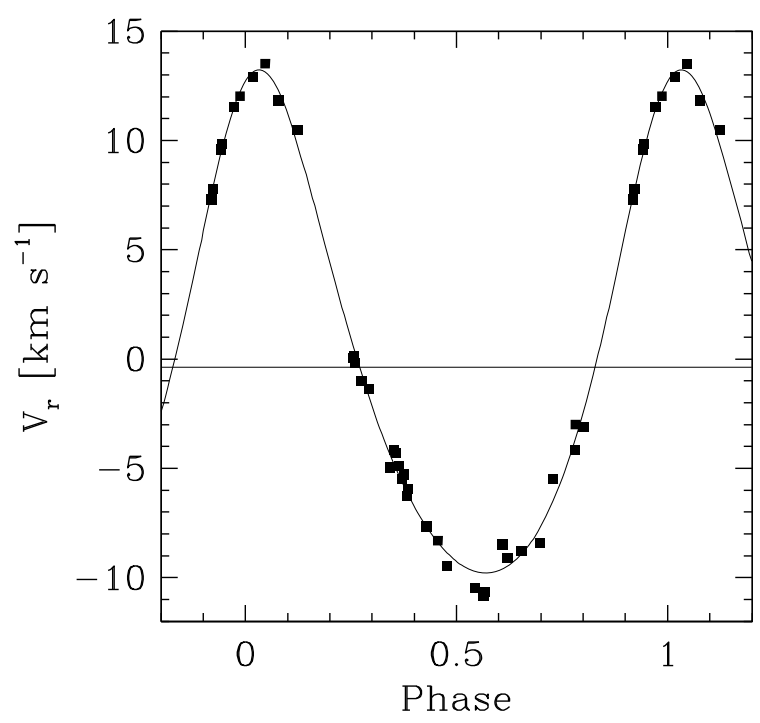

Fig. 5. Radial-velocity curve for the single-lined binary $\operatorname{Tr} 53$.

on, or close to, the single-star locus, while $\operatorname{Tr} 141$ is probably a photometric binary. Star \#397 lies some $12^{\circ}$ away from the cluster centre.

Ford et al. (2001) concluded that the other stars $\left(\mathrm{BD}+16^{\circ} 2505,+25^{\circ} 2631,+36^{\circ} 2312\right.$ and TYC 2534-1715-1) are non-members according to their radial velocities and absence of LiI lines at $6708 \AA$, although their $V, B-V$ photometry would locate them within the MS band. It would be useful to obtain additional radial velocities of $\mathrm{BD}+16^{\circ} 2505$ and $+25^{\circ} 2631$ to be sure that they are not binaries and settle more definitively their membership status.

\section{Am stars}

Five Am stars and one Ap ( $\operatorname{Tr} 146)$ were observed with CORAVEL. Tr 144 and 145 are both spectroscopic binaries with periods of 11.7 and 68.3 respectively (Abt \& Willmarth 1999).

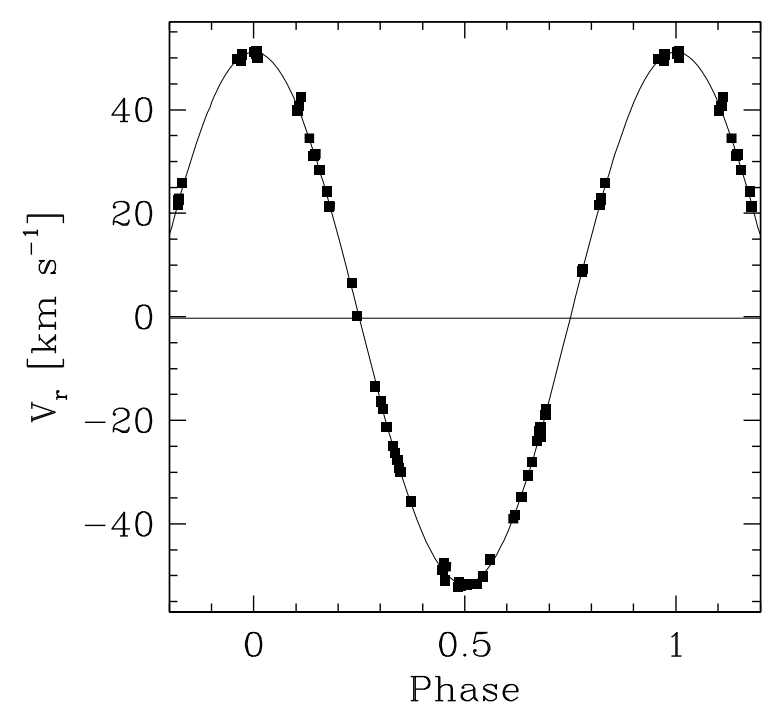

Fig. 6. Radial-velocity curve for the single-lined binary $\operatorname{Tr} 97$.

Table 5. Additional cluster members.

\begin{tabular}{llrrcl}
\hline \hline No. & Ident. & \multicolumn{1}{c}{$V$} & $B-V$ & $V_{\mathrm{r}}$ & Source \\
\hline 141 & BD $+29^{\circ} 2290$ & 9.72 & 0.71 & +0.6 & Ford et al. (2001) \\
385 & BD $+21^{\circ} 2514$ & 10.12 & 0.75 & -0.6 & Ford et al. (2001) \\
388 & HD 111154 & 8.40 & 0.56 & +0.6 & Griffin (2001) \\
392 & BD $+28^{\circ} 2119$ & 10.50 & 0.79 & +2.0 & Ford et al. (2001) \\
394 & HD 114400 & 9.59 & 0.61 & -1.7 & Ford et al. (2001) \\
397 & BD $+38^{\circ} 2436$ & 9.10 & 0.54 & -2.0 & Ford et al. (2001) \\
\hline
\end{tabular}

Mean radial velocities are given in Table 6. The orbital elements for $\operatorname{Tr} 144$ and 145 given in Table 7 confirm the previous ones. The radial-velocity curves are displayed in Figs. 15 and 16. Spectral types in Table 6 are from Gray \& Garrison (1989), and from Abt \& Cardona (1984) for Tr 146. Individual observations are given in Table 8. The complete data set is available in electronic form at the CDS. 


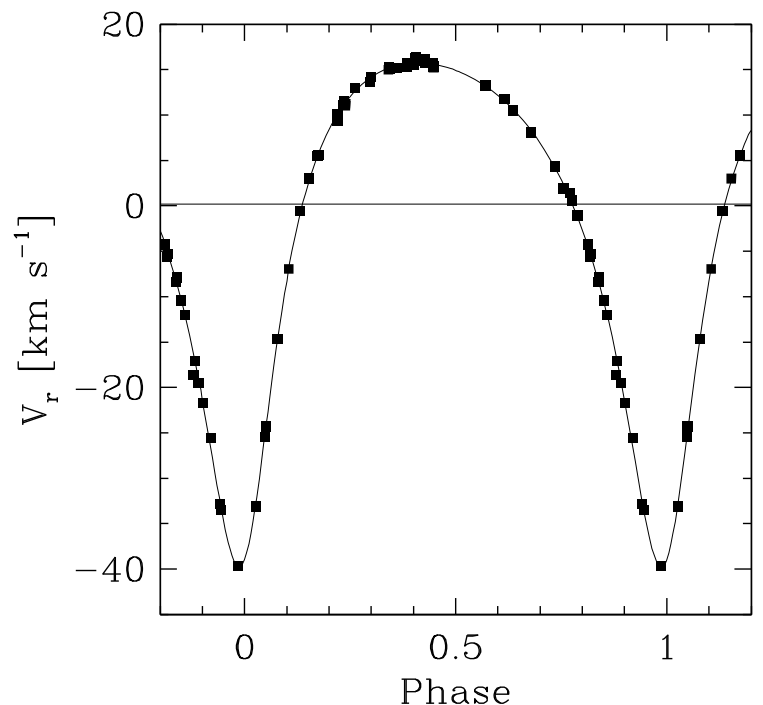

Fig. 7. Radial-velocity curve for the single-lined binary $\operatorname{Tr} 102$.

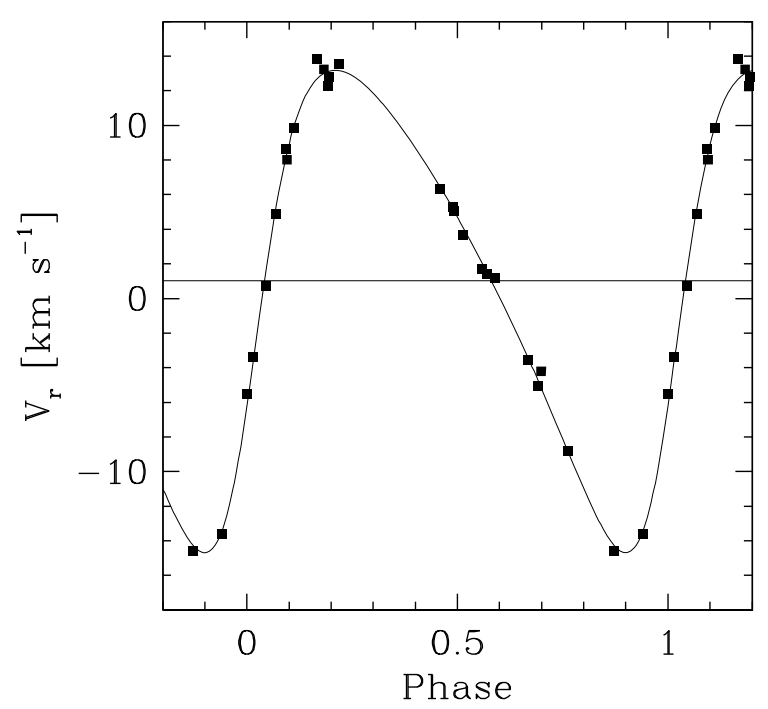

Fig. 8. Radial-velocity curve for the single-lined binary $\operatorname{Tr} 120$.

The other stars classified as Am did not vary in radial velocity. Star Tr 62 was observed 13 times between December 1979 and December 1997 and no variation was found. Similarily, $\operatorname{Tr} 183$ was measured 16 times over the same interval of time and no variation was seen. While $\operatorname{Tr} 62$ is clearly an Am star, $\operatorname{Tr} 183$ may be either a mild Am, with only a deficiency of $\mathrm{Ca}$ : $\mathrm{SP}(\mathrm{K}, \mathrm{H}$, M) = A5-A7-A7 (Abt \& Morrell 1995) or a normal star, classified A3 IV-Vs by Gray \& Garrison (1989) and A5 III by Cowley et al. (1969). Tr 139 is probably also constant, although the rotation produces larger correlation functions and hence less precise radial velocities. At least two Am stars, Tr 62 and Tr 139, are not spectroscopic binaries, which confirms the conclusions reached by Conti \& Barker (1973).

\section{Discussion}

Among the 74 stars observed, 38 turned out to be simply field stars, i.e. non-members. This high fraction confirms the difficulty of finding new bona fide members although the photometry of many of these candidates located them close together on the single-star locus or within the main-sequence band.

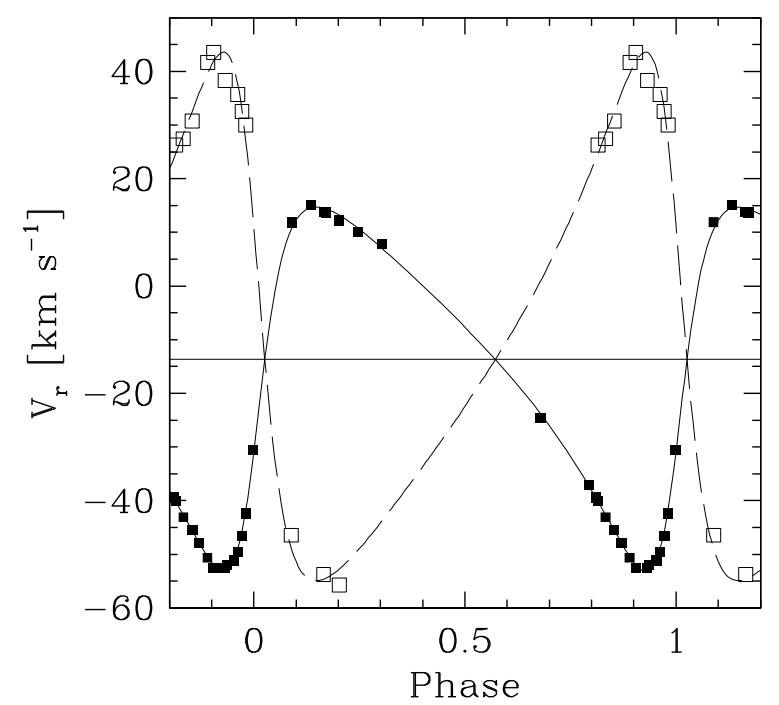

Fig. 9. Radial-velocity curve for the double-lined binary $\operatorname{Tr} 147$.

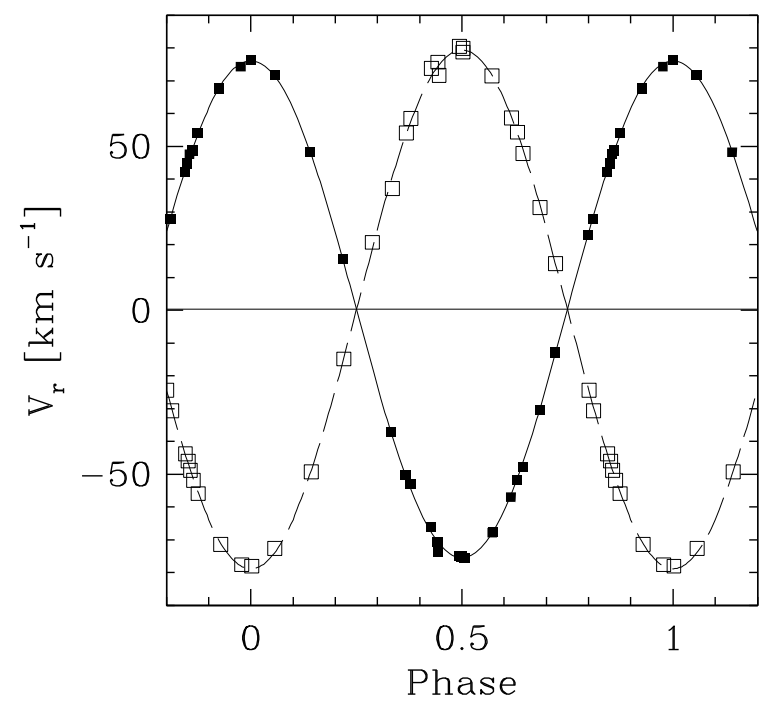

Fig. 10. Radial-velocity curve for the double-lined binary $\operatorname{Tr} 150$.

Table 6. Mean radial velocities of the Am and Ap stars.

\begin{tabular}{|c|c|c|c|c|c|c|c|c|c|}
\hline No. & MK type & $V_{r}$ & Eps & $n$ & $\Delta T$ & $P\left(\chi^{2}\right)$ & $V \sin i$ & Err & Notes \\
\hline 62 & $\mathrm{~A} 5 \mathrm{~m}$ & +1.28 & 0.17 & 13 & 6585 & 0.484 & 14.1 & 0.3 & $\mathrm{C}$ \\
\hline 139 & $\mathrm{~A} 5 \mathrm{~m}$ & +2.28 & 1.08 & 6 & 1803 & 5 & 38.4 & 3.8 & $\mathrm{Co}$ \\
\hline 144 & $\mathrm{~A} 7 \mathrm{~m}$ & -0.36 & 0.10 & 28 & 5166 & 0.000 & 8.2 & 0.3 & SB1O \\
\hline 145 & $\mathrm{~A} 2 \mathrm{~m}$ & +0.05 & 0.44 & 17 & 5166 & 0.000 & 20.1 & 2.0 & SB1O \\
\hline 146 & $\mathrm{~A} 1 \mathrm{Vp}$ & +3.79 & 1.89 & 2 & 26 & 0.210 & 26.8 & 2.7 & Const. \\
\hline 183 & A3IV-Vs & +1.62 & 0.35 & 16 & 6582 & 0.018 & 14.7 & 0.7 & Const. \\
\hline
\end{tabular}

One fundamental aspect in the search for detection of new members is the area in which members are looked for. The fact that star \#397 (BD $+38^{\circ} 2436$ ) lies some $12^{\circ}$ from the cluster centre and \#395 (BD $\left.+35^{\circ} 2278\right)$ at some $9^{\circ}$ seems to indicate that the cluster dimension is much larger than usually accepted. These two objects could be considered as belonging to the cluster corona, although they could be runaway stars, still sharing the same motion as the bulk of the cluster stars. This part of the surface has not been much investigated so far. Depending on the real radial distribution of the stars in the Coma Berenices cluster, a sizeable fraction of candidates could be located more than $5^{\circ}$ away from the cluster centre. The existence of such a population 


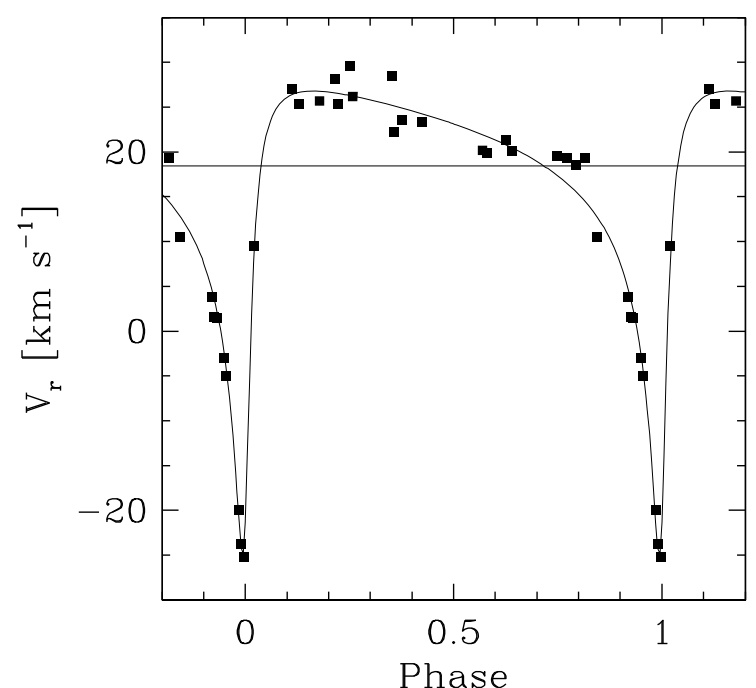

Fig. 11. Radial-velocity curve for the single-lined binary Malm 25.055.

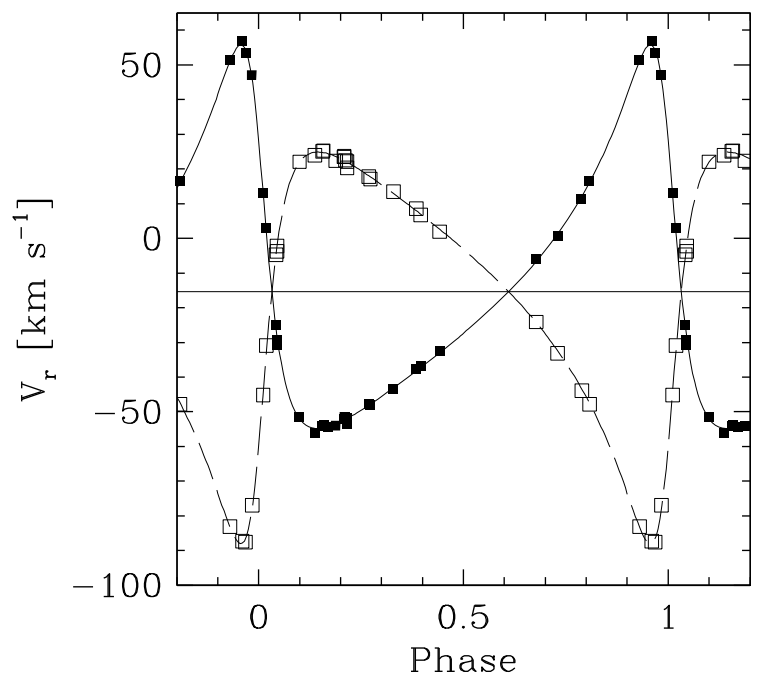

Fig. 12. Radial-velocity curve for the double-lined binary Malm 28.351.

of members in cluster coronae was proved by the CORAVEL radial-velocity surveys of the Pleiades (Rosvick et al. 1992; Mermilliod et al. 1997) and Praesepe (Mermilliod et al. 1990).

\section{Conclusions}

The radial-velocity survey of solar-type dwarfs in the Coma Berenices open cluster allowed us to confirm the membership of the 19 stars already selected by Trumpler (1938), and of 4 additional stars for which he did not obtain radial velocities. Orbits were determined for the 6 binary members presently known and for 4 double-lined binary non-members. At least one binary, Tr 150 with a short period $P=3.55$, rotates faster than the single stars of similar colours. The contrast is apparent because of the low rotation of the other solar-type members.

Coma Berenices seems to be the first well-studied nearby open cluster with a poorly-populated main sequence. Indeed, most studies performed so far have not succeeded in identifying a significant population of $\mathrm{K}$ - and $\mathrm{M}$ - type members, as observed in most other nearby open clusters. Lower-main-sequence stars, usually fainter than the limits of the available $U B V$ photoelectric photometry, were identified in other nearby clusters by X-ray

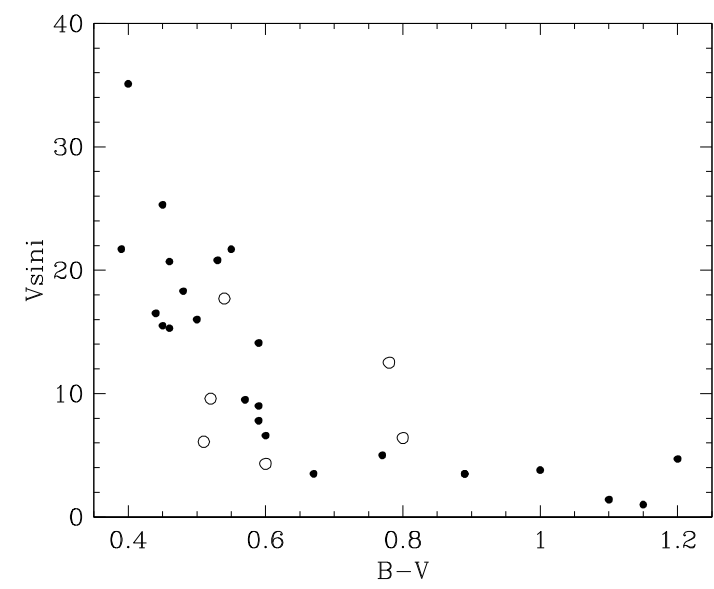

Fig. 13. Distribution of the projected rotational velocities as a function of the $B-V$ colours. The filled circles represent single stars and open circles, binaries.

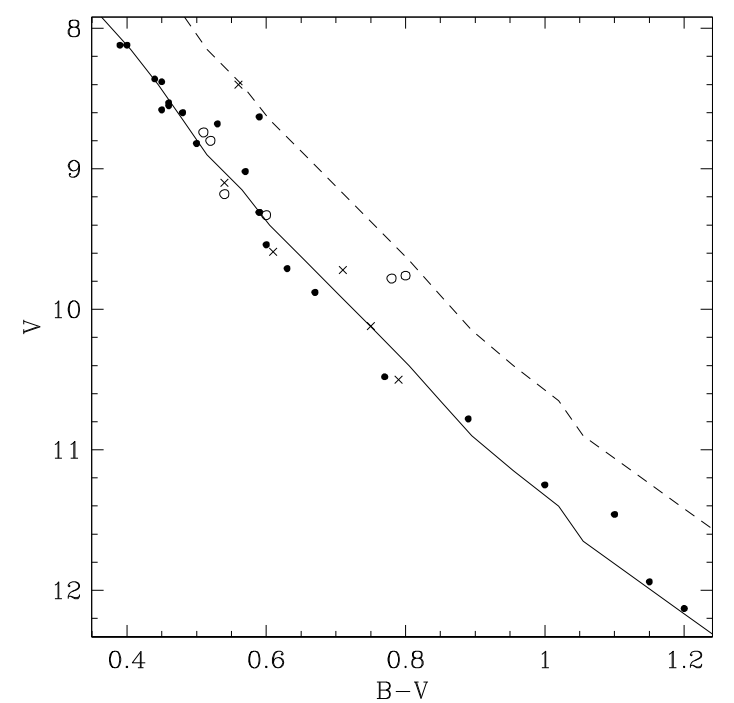

Fig. 14. Colour-magnitude diagram for the cluster members. The lower sequence is the ZAMS, and the upper one, the upper limit for binaries. The filled circles represent single stars and open circles, binaries, and crosses, five stars from Ford et al. (2001).

Table 7. Orbital elements of two Am stars.

\begin{tabular}{lcc}
\hline \hline No. & $\operatorname{Tr} 144$ & $\operatorname{Tr} 145$ \\
\hline$P[\mathrm{~d}]$ & 11.787023 & 68.252 \\
& 0.000034 & 0.022 \\
{$[\mathrm{HJD}]$} & 49987.406 & 49967.7 \\
& 0.006 & 1.3 \\
$V_{\mathrm{o}}\left[\mathrm{km} \mathrm{s}^{-1}\right]$ & -0.36 & +0.05 \\
& 0.10 & 0.44 \\
$\mathrm{e}$ & 0.0349 & 0.287 \\
& 0.0034 & 0.037 \\
$\omega\left[^{\circ}\right]$ & 155.1 & 262.9 \\
& 5.6 & 7.6 \\
$\mathrm{~K}\left[\mathrm{~km} \mathrm{~s}^{-1}\right]$ & 40.61 & 13.54 \\
& 0.14 & 0.39 \\
$f(m)$ & 0.08185 & 0.0154 \\
& 0.00087 & 0.0013 \\
$a \sin i[\mathrm{Gm}]$ & 6.579 & 12.17 \\
& 0.023 & 0.35 \\
$\sigma(\mathrm{O}-\mathrm{C})\left[\mathrm{km} \mathrm{s}^{-1}\right]$ & 0.47 & 1.12 \\
$n_{\text {obs }}$ & 28 & 17 \\
\hline
\end{tabular}




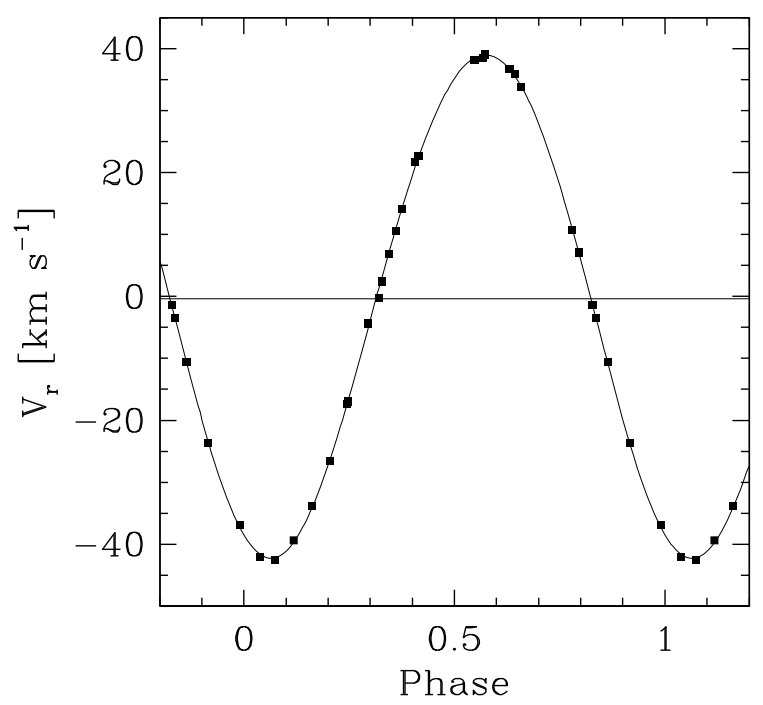

Fig. 15. Radial-velocity curve for the Am binary Tr 144.

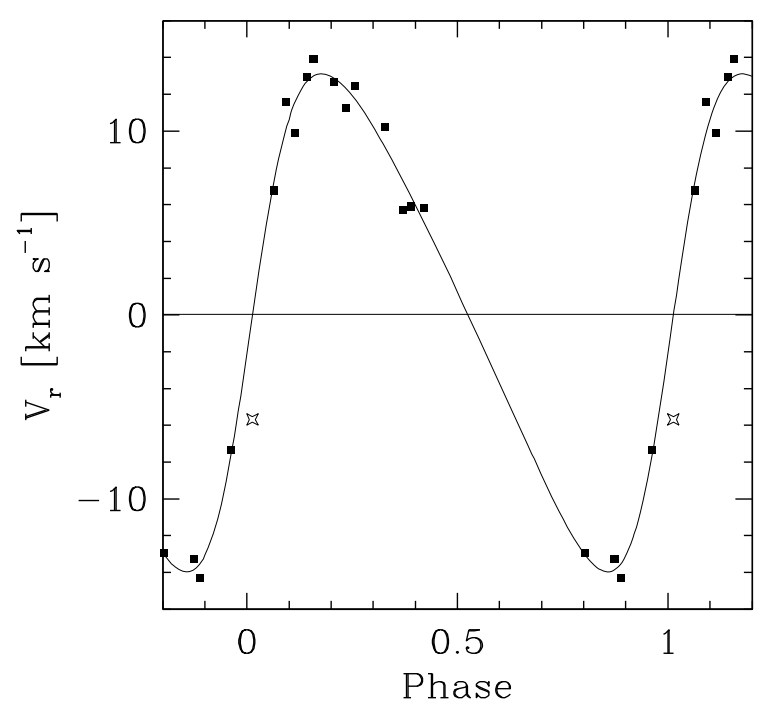

Fig. 16. Radial-velocity curve for the Am binary Tr 145 .

imaging. Although most solar-type dwarfs in Coma Ber do emit $\mathrm{X}$-rays, no additional stellar sources were found to be members of the Coma cluster. The candidates listed by Casewell et al. (2006) should be observed to settle their membership. However the very small proper motion and the radial velocity close to zero do not facilitate the selection of members. Depending on the results of these observations, confirming or not the membership of several of the candidates, Coma Ber may be a prominent example of dynamical evolution leading to star evaporation. However, the search area probably should be extended up to $10^{\circ}$ or even $12^{\circ}$ to identify corona members. The importance of its location at high latitude above the galactic plane should be evaluated to explain the paucity of low-mass members.
Table 8. Individual radial velocities.

\begin{tabular}{rcrr}
\hline \hline No. & HJD & Vr & pe \\
\hline 62 & 2444224.746 & +2.50 & 0.84 \\
62 & 2444227.723 & +1.57 & 0.73 \\
62 & 2444277.556 & +1.06 & 0.67 \\
62 & 2444287.609 & +1.21 & 0.68 \\
62 & 2444307.509 & +0.61 & 0.61 \\
\hline
\end{tabular}

Notes: Table 8 is available in its entirety from the CDS $\mathrm{ftp}$ archives at http: //cdsweb.u-strasbg.fr/cgi-bin/qcat?J/A+A/491/951

The study of the Coma Berenices cluster illustrates once again that kinematical data, in this case radial velocities, are of fundamental importance to determine with a high degree of reliability the membership of stars in nearby open clusters.

\section{References}

Abad, C., \& Vicente, B. 1999, A\&AS 136, 307 Abt, H. A., \& Cardona, O. 1984, ApJ, 276, 266 Abt, H. A., \& Morrell, N. I. 1995, ApJS, 99, 135 Abt, H. A., \& Willmarth, D. W. 1999, ApJ, 521, 682 Argue, A. N. 1963, MNRAS 127, 97

Argue, A. N., \& Kenworthy, C. M. 1969, MNRAS, 146, 479 Artyukhina, N. M. 1955, Tr. Gos. Astr. Inst. Sternberga, 26, 63 Baranne, A., Mayor, M., \& Poncet, J.-L. 1979, Vistas Astron., 23, 279 Baranne, A., Queloz, D., Mayor, M., et al. 1996, A\&AS, 119, 373 Benz, W., \& Mayor, M. 1984, A\&A, 138, 183

Bounatiro, L. 1993, A\&AS, 100, 531 Casewell, S. L., Jameson, R. F., \& Dobbie, P. D. 2006, MNRAS, 365, 447 Conti, P. S., \& Barker, P. K. 1973, ApJ, 186, 185

Cowley, A. P., Cowley, C., Jaschek, M., \& Jaschek, C. 1969, AJ, 74, 375 de Luca, E. E., \& Weis, E. W. 1981, PASP, 93, 32

Ford, A., Jeffries, R. D., James, D. J., \& Barnes, J. R. 2001, A\&A, 369, 871 Friel, E. D., \& Boesgaard, A. M. 1992, ApJ, 387, 170

García López, R. J., Randich, S., Zapatero Osorio, M. R., \& Pallavicini, R. 2000, A\&A, 363, 958

Gebran, M., Monier, R., \& Richerd, O. 2008, A\&A, 479, 189

Glushkova, E. 1993, priv. communication

Gratton, R. 2000, ASP Conf. Ser., 198, 225

Gray, R. O., \& Garrison, R. F. 1989, ApJS, 70, 623

Griffin, R. F. 2001, JAA, 22, 121

Harper, W. E. 1927, PDAO III, 315

Jeffries, R. D. 1999, MNRAS, 304, 821

Johnson, H. L., \& Knuckles, C. F. 1955, ApJ, 122, 209

Kraft, R. P. 1965, ApJ, 142, 681

Kraus, A. L., \& Hillenbrand, L. A. 2007, AJ, 139, 2340

Malmquist, K. G. 1927, Lunds Medd. II, 37

Malmquist, K. G. 1936, Stockholms Obs. Ann., 12, 7

Mermilliod, J.-C., Weis, E. W., Duquennoy, A., \& Mayor, M. 1990, A\&A, 235, 114

Mermilliod, J.-C., Bratschi, P., \& Mayor, M. 1997, A\&A, 320, 74

Mermilliod, J.-C., Udry, S., \& Mayor, M. 2008, in prep.

Odenkirchen, M., Soubiran, C., \& Colin, J. 1998, New Astron., 3, 583

Olsen, E. 1984, private communication

Randich, S., Schmitt, J. H. M. M., \& Prosser, C. 1996, A\&A, 313, 815

Rosvick, J., Mermilliod, J.-C., \& Mayor, M. 1992, A\&A, 255, 130

Trumpler, R. J. 1938, Lick Obs. Bull., 18, 167

Udry, S., Mayor, M., \& Queloz, D. 1999, in Precise Stellar Radial Velocities (IAU Colloq. 170), ed. J. B. Hearnshaw, \& C. D. Scarfe, ASP Conf. Ser., 185, 367

Upgren, A. R., Jr 1962, AJ, 67, 37 